\title{
market place
}

\section{Effective bone regeneration}
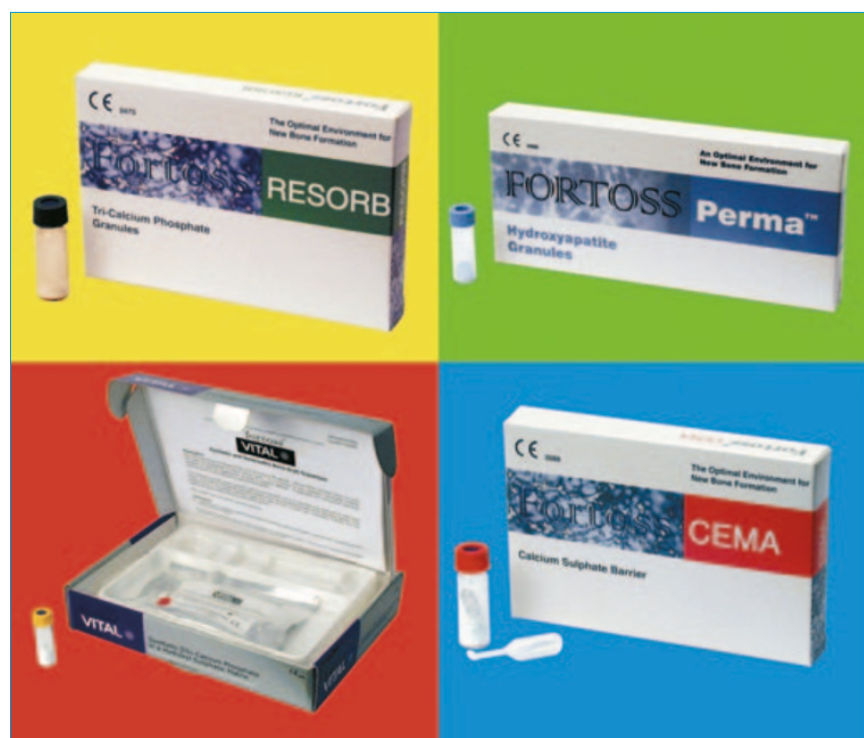

Fortoss is a range of bone regeneration products available from The Dental Directory. There are four products in total - Resorb, Cema, Perma and Vital. Resorb is a versatile material that can be used around immediate implants, sinuses, dehiscences and extraction sockets where implants are indicated. Cema is an easy to use resorbable membrane that is applied as a paste and sets under the soft tissues and is compatible with any augmentation material. Perma can be used for maintaining ridge height and width after extraction or to build bone lost to peri-implantitis. Lastly, there's Fortoss Vital, a synthetic bone regenerative that mimics the mineral phase of human bone and can be used for periodontal work or surgical endodontics as well as around implants, sinuses, dehiscences and extraction pockets.

\section{Popular}

\section{instrument sets}

BDSI Ltd can provide a range of instruments and small equipment such as the new vacuum autoclave Prestige C3 advanced model and the new Prolux LED curing light which is available in three colours.

\section{New education programme}

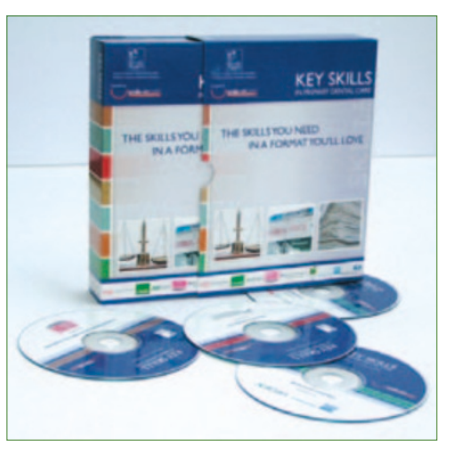

Smile-on's Key Skills in Primary Care is a CD ROM-based education programme using scenario-based learning. It covers a significant spectrum of practice issues that can be applied in dental practices.

Seven specialist subject matters are covered, one such area is patient confidentiality in record keeping, and suggests measures to ensure confidentiality. These measures take the common sense approach, and reinforce practice protocols such as ensuring all filing cabinets are clearly labelled and secured, and computer monitors and patients' records (including radiographs, etc) are kept out of the view of the general public.

\section{New additions to range}

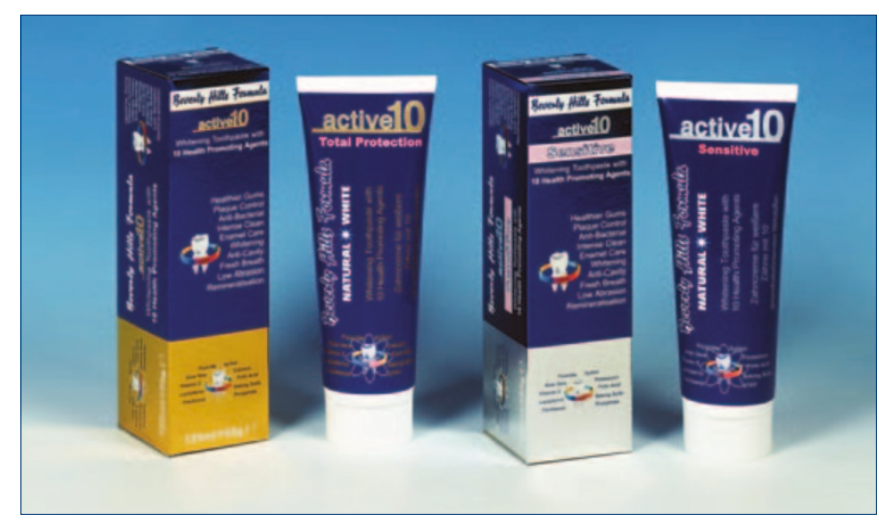

Beverly Hills Formula have launched the new Active 10 products range (available in two variants: Total Protection and Sensitive) to add to its existing range of teeth whitening toothpastes.

As its name suggests, Active 10 is a formula that benefits from ten health promoting agents which not only protect teeth and gums and promote whitening, but also improve and maintain a healthy mouth. The toothpaste offers total protection, whitening and intense cleaning with plaque control, low abrasion, gum protection, enamel care and remineralisation. It also has an anti-inflammatory action, anti-cavity protection and promotes fresh breath. 